\title{
Explosive transitions induced by interdependent contagion-consensus dynamics in multiplex networks
}

\author{
D. Soriano-Paños, ${ }^{1,2}$ Q. Guo, ${ }^{3,4, *}$ V. Latora, ${ }^{5,6,7, \dagger}$ and J. Gómez-Gardeñes ${ }^{1,2, \$}$ \\ ${ }^{1}$ GOTHAM Laboratory, Institute for Biocomputation and Physics of Complex Systems (BIFI), University of Zaragoza, 50018 Zaragoza, Spain \\ ${ }^{2}$ Departamento de Física de la Materia Condensada, Universidad de Zaragoza, 50009 Zaragoza, Spain \\ ${ }^{3}$ School of Mathematics and Systems Science, Beihang University \& Key Laboratory of Mathematics Informatics Behavioral Semantics \\ (LMIB), Beijing 100191, China \\ ${ }^{4}$ China Construction Bank, Beijing 100033, China \\ ${ }^{5}$ School of Mathematical Sciences, Queen Mary University of London, London E1 4NS, United Kingdom \\ ${ }^{6}$ Dipartimento di Fisica ed Astronomia, Università di Catania and INFN, Catania I-95123, Italy \\ ${ }^{7}$ Complexity Science Hub Vienna (CSHV), 1080 Vienna, Austria
}

(Received 20 December 2018; revised manuscript received 22 April 2019; published 25 June 2019)

\begin{abstract}
We introduce a model to study the interplay between information spreading and opinion formation in social systems. Our framework consists in a two-layer multiplex network where opinion dynamics takes place in one layer, while information spreads on the other one. The two dynamical processes are mutually coupled in such a way that the control parameters governing the dynamics of the node states at one layer depend on the dynamical states at the other layer. In particular, we consider the case in which consensus is favored by the common adoption of information, while information spreading is boosted between agents sharing similar opinions. Numerical simulations of the model point out that, when the coupling between the dynamics of the two layers is strong enough, a double explosive transition, i.e., a discontinuous transition both in consensus dynamics and in information spreading appears. Such explosive transitions lead to bi-stability regions in which the consensus-informed states and the disagreement-uninformed states are both stable solutions of the intertwined dynamics.
\end{abstract}

DOI: 10.1103/PhysRevE.99.062311

\section{INTRODUCTION}

The functioning of a wide range of complex systems in physics, biology, and social sciences is subject to collective phenomena such as the onset of synchronization [1], the emergence of norms and cooperation [2], or the diffusion of epidemics [3], among others. In the past two decades, a number of works have analyzed the role played by the structure of the networks governing the interactions among the constituents of a complex system in the emerging of its collective dynamics [4-8]. Our understanding of the fundamental mechanisms driving these phenomena is of utmost importance, as it provides a solid basis for modeling, predicting, and controlling real dynamical systems [9-11].

Recently, complexity and network science have moved one step forward in this direction by considering that, very frequently, the elements of many real complex systems are subject to different types of interactions at the same time. In some cases, the dynamical processes that occur simultaneously in a system depend on each others. Examples of the coexistence and non-trivial interdependence of two or more dynamical processes are very common in social systems and in the natural sciences. For instance, human prevention be-

\footnotetext{
*quantongg@buaa.edu.cn

†v.latora@qmul.ac.uk

† gardenes@unizar.es
}

haviors coexist and co-evolve with disease spreading [12-15], while different dynamical processes interact in neural systems and govern the structure-function relationships in the human brain [16].

The study of such coupled dynamical processes has been largely stimulated by the introduction of novel frameworks to deal with networks with many layers or networks of networks [17-20]. Multiplex networks are indeed the natural way to model the existence of different dynamical interactions among the same set of units [21-31].

In this work we introduce and study a model of two coevolving socially inspired processes: formation of opinions [32] and information spreading [33]. In our model the two dynamics are mutually coupled in such a way that the transmission of information from a spreader to a receiver is boosted when the neighbors of the latter share similar opinions [34]. In addition to this, the alignment of the opinion of an agent to those of her neighbors is fostered when such neighbors spread the voice simultaneously. The model, and in particular the adopted type of interdependence of the two processes, captures everyday life examples in which the use of technology or the adoption of new ideas by an individual happens in virtue of the consensus found among her acquaintances $[35,36]$ and, in turn, the common adoption of these novelties boosts the degree of homophily needed for the creation of social consensus [37,38]. The study of the model reveals how the interplay between opinion and spreading dynamics can dramatically alter the critical properties of the two dynamical 
processes, leading to abrupt onsets of epidemics and consensus. These explosive onsets are discontinuous transitions leading to the appearance of bistable regions where the multiplex network can switch between active to inactive dynamical phases triggered by small perturbations. It is remarkable that the explosive transitions that we observe in our model are not produced by any of the standard mechanisms at work in single networks [39], but they are the result of the coupling induced by the multiplex architecture.

The work is organized as follows. In Sec. II we introduce the model and discuss the rationale under the adopted dynamical coupling between layers. Then, in Sec. III, we illustrate, through numerical simulations of the model, the emergence of explosive transitions both in the consensus and in the contagion dynamics. In Sec. IV, we numerically explore the role that both topological and dynamical features of the model play on the emergence of these abrupt transitions. Finally, in Sec. V we summarize the main results of this work and we discuss possible future directions.

\section{MODEL OF INTERDEPENDENT DYNAMICS}

To describe the delicate interplay between information spreading and the formation of consensus in a social system, we introduce here a model in which the two processes take place at the two layers of a multiplex network with $M=2$ layers and are mutually coupled. We deal with a multiplex network following the assumption that there exists a oneto-one correspondence between nodes (the social agents) in different layers, so that each layer is composed by the same set of $N$ nodes. However, the topologies of the two layers can in general be different and are described by the adjacency matrices $A^{[1]}=\left\{a_{i j}^{[1]}\right\}$ and $A^{[2]}=\left\{a_{i j}^{[2]}\right\}$, respectively. These matrices are defined such that $a_{i j}^{[1]}=1\left(a_{i j}^{[2]}=1\right)$ if a link exists between nodes $i$ and $j$ in the first (second) layer, while $a_{i j}^{[1]}=0\left(a_{i j}^{[2]}=0\right)$ otherwise. We denote the degree of node $i$ in the first (second) layer as $k_{i}^{[1]}=\sum_{j=1}^{N} a_{i j}^{[1]}\left(k_{i}^{[2]}=\right.$ $\left.\sum_{j=1}^{N} a_{i j}^{[2]}\right)$.

Our model can be phrased in terms of a general formalism for interdependent dynamical networks proposed in Ref. [29]. If we denote, respectively, as $\quad \mathbf{x}(\mathbf{t})=\left\{x_{1}(t), x_{2}(t), \ldots, x_{N}(t)\right\} \in \mathfrak{R}^{N} \quad$ and $\quad \mathbf{y}(\mathbf{t})=$ $\left\{y_{1}(t), y_{2}(t), \ldots, y_{N}(t)\right\} \in \Re^{N}$ the states of the nodes at the two layers, then the evolution of the system can be written as

$$
\begin{aligned}
\dot{x}_{i} & =F_{\xi_{i}}\left(\mathbf{x}, A^{[1]}\right) \\
\dot{y}_{i} & =G_{\eta_{i}}\left(\mathbf{y}, A^{[2]}\right) \quad i=1,2, \ldots N,
\end{aligned}
$$

where the dynamics of state $x_{i}\left(y_{i}\right)$ of node $i$ in the first (second) layer is governed by a function $F_{\xi}\left(G_{\eta}\right)$ of the dynamical state $\mathbf{x}(\mathbf{y})$ and of the structure $A^{[1]}\left(A^{[2]}\right)$ of the first (second) layer. Notice that, following Ref. [29], functions $F_{\xi}$ and $G_{\eta}$ in our model are taken to be dependent on the parameters $\xi$ and $\eta$, and this is the key ingredient to connect the two dynamical processes. Namely, we assume that the parameter $\xi_{i}$ of function $F_{\xi_{i}}$ at the first layer is itself a function of time depending on the dynamical states $\left\{y_{j}(t)\right\}$ at the second layer of the neighbors $j$ of node $i$ at the first layer
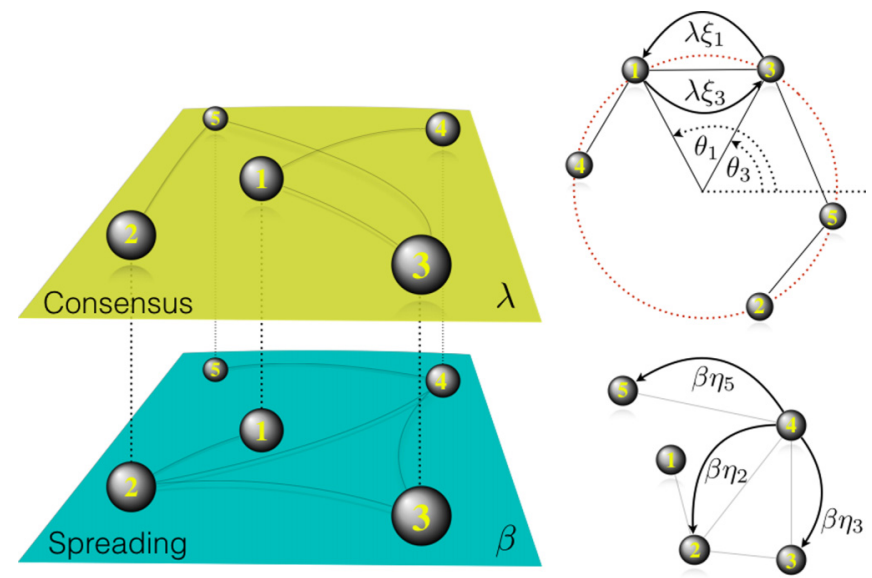

FIG. 1. Left: Schematic representation of our model on a multiplex network with $M=2$ layers and $N=5$ nodes. The first (top) layer accounts for the consensus dynamics, which is modeled by a Kuramoto model as in Eq. (3), whereas the second (bottom) layer describes the spreading of information according to the SIS model as in Eq. (4). Right: The coupling strength $\lambda$ between opinions (top) as well as the contagion rate $\beta$ (bottom) have been modified as in Eqs. (7) and (6), respectively, to mutually couple the synchronization process to the spreading of information.

$\left(a_{i j}^{[1]}=1\right)$. Analogously, the evolution of the parameter $\eta_{i}$ at the second layer depends on the states $\left\{x_{j}(t)\right\}$ at the first layer of the neighbors of node $i$ at the second layer $\left(a_{i j}^{[2]}=1\right)$. In this way, the system of Eq. (1) is completed by the following system of equations:

$$
\begin{aligned}
& \xi_{i}(t)=f\left(\left\{y_{j}(t) \mid a_{i j}^{[1]}=1\right\}\right) \\
& \eta_{i}(t)=g\left(\left\{x_{j}(t) \mid a_{i j}^{[2]}=1\right\}\right) \quad i=1,2, \ldots N,
\end{aligned}
$$

where $f$ and $g$ are two assigned functions.

As illustrated in Fig. 1, the first layer in our model accounts for the dynamics underlying the formation of consensus in a social system, while the second layer describes the contagion processes mimicking the spread of ideas/products. The dynamical state $x_{i}(t)$ of node $i$ at the first layer represents the opinion of individual $i$, described as a phase variable, i.e., $x_{i}(t)=\theta_{i}(t) \in[-\pi, \pi]$. The time evolution of $x_{i}(t)$ is modeled via the Kuramoto model of coupled phase-oscillators [40-42], so that the first set of equations in the system of Eq. (1) reads

$$
\dot{\theta}_{i}(t)=F_{\xi_{i}}\left(\theta, A^{[1]}\right)=\omega_{i}+\lambda \xi_{i}(t) \sum_{j=1}^{N} a_{i j}^{[1]} \sin \left[\theta_{j}(t)-\theta_{i}(t)\right],
$$

where $\omega_{i}$ is the natural frequency of node $i$. Notice that $\lambda$ is a global coupling strength, while the local coupling strength associated to node $i$ is modulated by the dynamical variable $\xi_{i}(t)$ that changes in time depending on the dynamics of the second layer, as sketched in Eq. (2), in a way that will be specified below.

The dynamical state $y_{i}(t)$ of node $i$ at the second layer represents the probability of node $i$ of being active as user/spreader of an idea, namely, $y_{i}(t)=p_{i}(t) \in[0,1]$. The 
time evolution of $p_{i}(t)$ is modeled through a susceptibleinfected-susceptible (SIS) model. In this way we identify susceptible (ignorant or uninformed) agents as those who do not have and transmit information, whereas the infected ones correspond to active users (informed and spreaders) who disseminate the information to the rest of the population. Under this framework, a susceptible node (a node in state $S$ ) with an infected neighbor can be infected by it at time $t$ through the process $S+I \rightarrow 2 I$ and becomes itself a spreader (state $I$ ) with a probability $\beta \eta_{i}(t)$. In addition, a spreader can return to its ignorant state through the process $I \rightarrow S$ with a probability $\mu$. Such a dynamics can be cast in the form of a Markov evolution for the probability $p_{i}(t)$ that a node $i$ is spreader at time $t$ as [43-45]

$$
\begin{aligned}
\dot{p}_{i}(t)= & -\mu p_{i}(t)+\left[1-p_{i}(t)\right] \\
& \times\left\{1-\prod_{j=1}^{N}\left[1-a_{i j}^{[2]} \beta \eta_{i}(t) p_{j}(t)\right]\right\} .
\end{aligned}
$$

Notice that, at variance with the usual SIS model, here the microscopic contagion probability $\beta \eta_{i}(t)$ of node $i$ may differ from node to node, and also change in time due to the presence of the time dependence in factor $\eta_{i}(t)$, in close analogy with the presence of the time-dependent factor $\xi_{i}(t)$ in the effective coupling of unit $i$ at the consensus layer.

To completely define our model we finally need to assign the time-dependent functions $\left\{\xi_{i}(t)\right\}$ and $\left\{\eta_{i}(t)\right\}$ that mutually couple the consensus dynamics and the process of contagion as sketched in Eq. (2). To define $\eta_{i}(t)$, we need to capture the influence that consensus at layer 1 has on the contagion dynamics at layer 2 . With this purpose we evaluate the local degree of consensus $r_{i}(t)$ around node $i$ at time $t$ by considering the values of $\theta_{j}(t)$ in the neighborhood of node $i$. Notice, however, that the neighbors of node $i$ are taken in the second layer, where information spreading takes place. This is because it is the consensus among potential spreaders that facilitates the transmission of ideas. We therefore use the adjacency matrix $\left\{a_{i j}^{[2]}\right\}$ to construct the neighborhood of node $i$. The local degree of consensus of node $i$ is defined as the modulus of the complex function:

$$
r_{i}(t) e^{i \psi_{i}(t)}=\frac{1}{k_{i}^{[2]}} \sum_{j=1}^{N} a_{i j}^{[2]} e^{i \theta_{j}(t)},
$$

so that we get $r_{i} \simeq 0$ in the absence of local consensus and $r_{i}=1$, otherwise. Once evaluated $r_{i}(t)$, we can write the second of Eq. (2) as

$$
\eta_{i}(t)=\frac{1}{1+\exp \left\{-\alpha\left[r_{i}(t)-r^{*}\right]\right\}} .
$$

The use of the Fermi function with a tuning parameter $\alpha>0$ implies that, for large enough values of $\alpha$, when $r_{i}(t) \rightarrow 0$, i.e., when the local consensus around $i$ is small, the contagion probability toward $i, \beta \eta_{i}(t)$, tends to 0 . However, when consensus among the neighbors of $i$ increases, their influence over $i$ also grows, approaching $\beta$ as $r_{i}(t) \rightarrow 1$. In this way the value $r^{*}$ acts as a threshold, so that for $r_{i}(t)>r^{*}\left(r_{i}(t)<r^{*}\right)$ we have $\eta_{i}(t)>0.5\left(\eta_{i}(t)<0.5\right)$. For the sake of simplicity, in the following we fix $r^{*}=0.5$.
Last, we model the influence that the contagion dynamics of layer 2 has on the formation of consensus at layer 1 . To this aim, the node-depending coupling constant $\lambda \xi_{i}(t)$ of the Kuramoto model at layer 1 is chosen to be dependent on the number of spreaders around node $i$ at layer 1. Specifically, $\xi_{i}(t)$ is defined as the fraction of spreaders among the neighbors of node $i$ in layer 1, so that the first of Eqs. (2) reads

$$
\xi_{i}(t)=\frac{\sum_{j=1}^{N} a_{i j}^{[1]} p_{j}(t)}{k_{i}^{[1]}} .
$$

Notice that this time we have made use of the adjacency matrix $\left\{a_{i j}^{[1]}\right\}$ to construct the neighborhood of node $i$.

Summing up, in our model the state $\left[\theta_{i}(t), p_{i}(t)\right]$ of each node $i$, with $i=1,2, \ldots, N$, evolves in time as in Eqs. (3) and (4), where the two parameters $\xi_{i}$ and $\eta_{i}$ depend in turn on the state $\left[\theta_{i}(t), p_{i}(t)\right]$ as in Eqs. (7) and (6), mutually coupling the two dynamical processes. Notice that in this way both the infection probability $\beta \eta_{i}$ and the Kuramoto coupling strength $\lambda \xi_{i}$ of a node $i$ are obtained by taking average over the neighbors in the layer that governs the corresponding dynamics, i.e., layer 1 for $\xi_{i}$ and layer 2 for $\eta_{i}$. However, the averaged dynamical quantities correspond to the node states at the other layer, i.e., the phases for $\eta_{i}$ and the probabilities of being infected for $\xi_{i}$, thus closing the feedback loop between spreading and consensus dynamics. The way the interdependence between these two processes has been modelled follows, as discussed above, the rationale that the existence of consensus facilitates the adoption of ideas and that it is the simultaneous spread of ideas that fosters the alignment of opinions.

\section{RESULTS}

To characterize the effects of the interplay between spreading and consensus dynamics, we explore the dynamical behavior of our coevolving model mainly focusing on the onset of synchronization and on the appearance of an endemic state. To this aim, we start by infecting a small fraction $\rho$ of agents and by initially setting the oscillator phases $\theta_{i}$ at random within a range $\theta_{i} \in(-\pi, \pi]$. The natural frequencies of oscillators $\left\{w_{i}\right\}$ are also randomly chosen within $w_{i} \in[-0.5,0.5]$. The particular values of these individual properties of nodes remain the same for all the numerical experiments. This way, we avoid the stochastic noise inherent to a random assignment of the initial conditions. We take $\lambda$ and $\beta$ as the natural control parameters for Kuramoto and SIS dynamics, respectively. The order parameters are also the usual ones for both dynamical systems. Namely, the degree of global consensus is measured by using the Kuramoto order parameter $r$ defined by the complex number:

$$
r(t) e^{i \psi(t)}=\frac{1}{N} \sum_{j=1}^{N} e^{i \theta_{j}(t)},
$$

which represents the centroid of all oscillators placed on the complex unit circle. In its turn, for the SIS model we monitor 

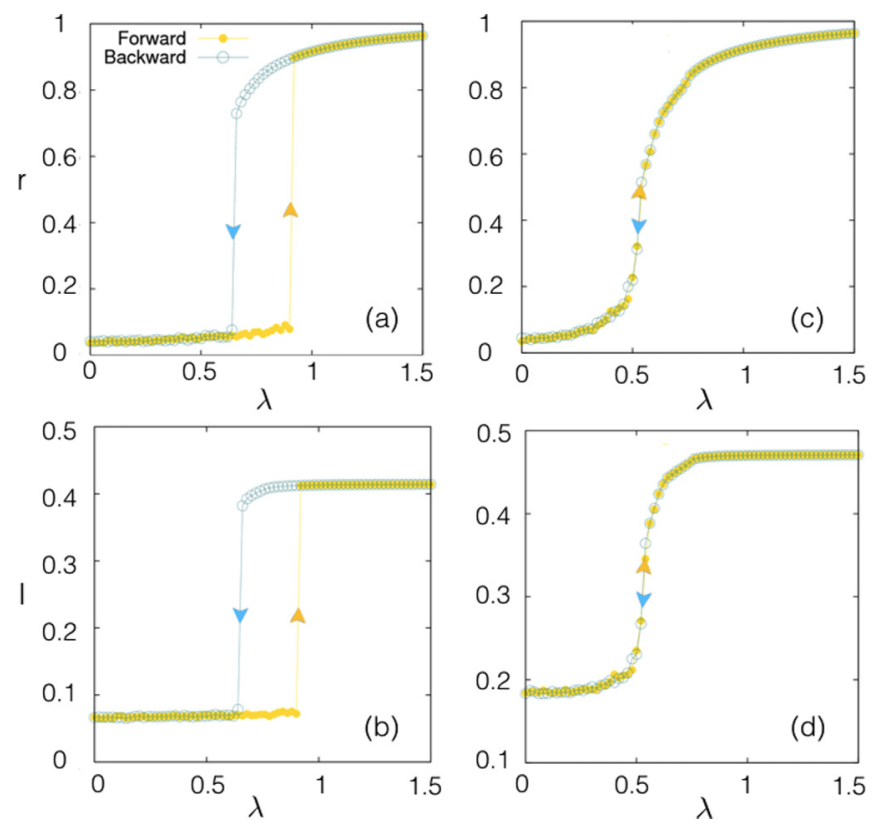

FIG. 2. Average global consensus $r$ (top) and fraction of information spreaders $I$ (bottom) as a function of the coupling constant $\lambda$ for the SF-ER multiplex configuration. These order parameters have been computed adiabatically by increasing the value of the coupling constant $\lambda$ (Forward) from $\lambda=0$ or by decreasing it (Backward) from $\lambda=1.5$. The contagion rate values used are for (a) and (b) $\beta=$ 0.70 and for (c) and (d) $\beta=1$. The rest of the model parameters are set to $\alpha=10$ and $\mu=1.0$. All the layers are composed by $N=500$ nodes with average degree $\langle k\rangle=4$. The SF network follows a power law distribution with exponent $\gamma=3$.

the evolution of the fraction of infected individuals:

$$
I(t)=\frac{1}{N} \sum_{j=1}^{N} p_{j}(t) .
$$

As usual, the order parameters, $r$ and $I$, are measured by making a time average of $r(t)$ and $I(t)$, once the stationary regime of the dynamics is reached. To reach this stationary state, we integrate Eq. (3) by using the fourth-order RungeKutta method and Eq. (4) using an Euler method, both with time steps $\delta t=0.01$.

The networks used to build the multiplex configurations are random Erdös-Renyi (ER) and scale-free (SF) networks with $N=500$ nodes and average degree $\langle k\rangle \simeq 4$. In particular, the SF networks are constructed according to the Barabási-Albert method [46], so the degree distribution follows a power law with exponent $\gamma=3$. The use of these two topologies allows us to study the role of degree heterogeneity in the evolution of consensus and spreading dynamics. As anticipated above, we denote the multiplex considering that the first layer contains consensus dynamics whereas information spreading takes place on top of the second one.

In Fig. 2, we have computed the diagrams for global consensus and fraction of infected people using a SF-ER multiplex by keeping fixed the contagion probability, $\beta$, in the ER layer and varying the consensus coupling in the SF one. To this aim, we have computed the forward (increasing $\lambda$ ) and backward (decreasing $\lambda$ ) diagrams. Figures 2(a), 2(b)
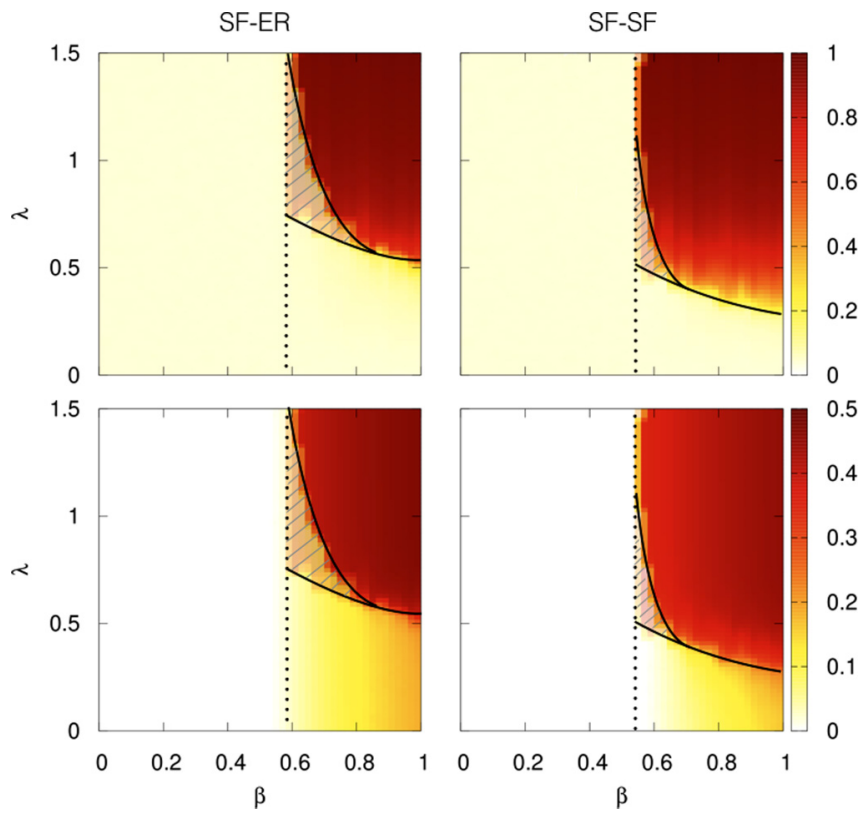

FIG. 3. Diagrams of the average global consensus $r$ (top) and of the fraction of spreaders nodes $I$ (bottom) as a function of the infectivity $\beta$ and the coupling parameter $\lambda$ for SF-ER (left) and SF-SF (right) multiplex networks. The color code encodes the magnitudes of the two order parameters. The striped regions in the panels highlight the parameter region $(\lambda, \beta)$, where hysteresis cycles appear due to the coexistence of two stable solutions: total consensus-disagreement in the synchronization layer and activeinactive spreaders at the information spreading layer. The black solid lines denote the critical values of the coupling $\lambda$ separating the two solutions. All the layers consists of $N=500$ nodes with average degree $\langle k\rangle=4$. The SF networks follow a power law distribution with exponent $\gamma=3$.

and 2(c), 2(d) show drastically different transitions. On one hand, Figs. 2(a) and 2(b), which correspond to $\beta=0.70$, show an abrupt transition both for the degree of consensus and the fraction of spreaders. These diagrams are characterized by the existence of regions of bistability where the solutions corresponding to absence of global consensus and information spreaders coexist with those displaying macroscopic coherence and spreading. On the other hand, Figs. 2(c) and 2(d), corresponding to $\beta=1$, show a smooth and continuous transition, i.e., the expected onset from the usual Kuramoto and SIS models. As we show below the particular type, smooth of explosive, of transition depends on the multiplex configuration and on the $\beta$ value.

To have a broader picture about the phenomenon described above, in Fig. 3 we represent the diagrams for global consensus $r$ (top row) and fraction of spreaders $I$ (bottom row) as a function of both $\beta$ and $\lambda$ for SF-ER (left panels) and SF-SF (right panels) multiplexes. At first sight, for both topologies, we observe that below a critical value $\beta_{c}$, represented with dashed points, the single stable solution is the absence of global consensus and information spreaders. Above this threshold, we can find different stable solutions depending on the Kuramoto coupling constant $\lambda$. Namely, for small values of $\lambda$, the stable solution is the absence of global consensus and the presence of a small fraction of spreaders $I$ which depends 
on the value of the contagion rate $\beta$. This constitutes a surprising result, since one would expect that, for large values of $\alpha$, the absence of global consensus prevents the diffusion of information. However, the finite-size effects associated to the reduced set of neighbors of each node in the spreading layer leads to the emergence of spreaders. In particular, despite the fact that there not exist a global shared opinion in the network for the non-synchronized regime, each agent observes some degree of local consensus which fosters the spread of ideas in this model. In the next section, we deeply study this region of the phase diagram unveiling the role that dynamical or topological features of the intertwined dynamics play on the diffusion of information despite the absence of consensus.

For larger values of $\lambda$, the type of transition observed depends on the value of $\beta$. In particular, for $\beta$ values close to the diffusion threshold, the number of spreaders in the non-synchronized regime is very small, so an abrupt transition takes place toward the state of global consensus and the existence of a macroscopic set of spreaders. Interestingly, these abrupt transitions incorporate bi-stability regions (see striped areas in Fig. 3) where the coexistence of two solutions (corresponding to large and small order parameters) explains the hysteresis cycles shown in Fig. 2. However, for large values of $\beta$, a macroscopic set of spreaders already exists in the non-synchronized regime, thus giving rise to a continuous transition in which both consensus and number of spreaders continuously grow while increasing the coupling constant $\lambda$.

At this point, we can understand the role that each process plays on the intertwined dynamics. It becomes clear that the epidemics behaves as the limiting process, for the emergence of consensus requires the existence of active spreaders but no viceversa. In its turn, the synchronization dynamics, monitored by the coupling constant $\lambda$, behaves as an external force which drives the system from a practically inactive phase to an active one.

Once described the diagrams in the $(\beta, \alpha)$ plane let us identify the main differences between SF-ER and SF-SF multiplexes. By comparing panels corresponding to the SFER configuration and those corresponding to the SF-SF one in Fig. 3, it is clear that the value of the critical coupling $\lambda_{c}$ separating the regions corresponding to the absence and presence of global consensus is lower for the SF-SF configuration than for the SF-ER one. To explain this, we must take into account that the degree distributions of both layers in the SF-SF configuration are positively correlated, so that hubs promote the interplay between consensus dynamics and information spreading, thus anticipating the explosive onsets. Another difference between both multiplexes, is that the bistable regime is hindered in the SF-SF configuration with respect to the SF-ER one.

\section{NUMERICAL STUDY OF THE INTERPLAY BETWEEN TOPOLOGY AND DYNAMICS}

In contrast to the differences discussed in the previous section regarding the phase diagrams of SF-ER and SF-SF multiplexes, the value of the diffusion threshold $\beta_{c}$ is roughly the same in both cases. This is an unexpected result, since the presence of hubs in heterogeneous networks boosts spreading phenomena in the vast majority of dynamical models.

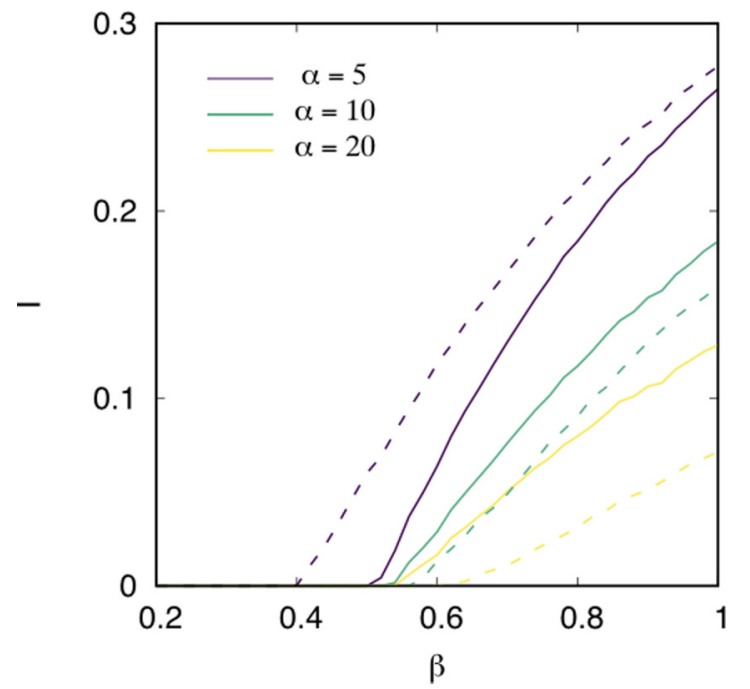

FIG. 4. Fraction of spreaders in the nonsynchronized regime $(\lambda=0)$ as a function of the transmissibility $\beta$ for both SF-ER (solid lines) and SF-SF configurations (dashed lines). The line color denotes the value of the social pressure $\alpha$. Regarding the underlying topologies, all layers are composed by $N=500$ nodes with average degree $\langle k\rangle=4$. The degree distribution of the SF layer follows a power law distribution with exponent $\gamma=3$.

However, in our model of intertwined dynamics, the probability that highly connected nodes diffuse information is also affected by the degree of consensus among their acquaintances. In this sense, the more neighbors agents have, the wider is the set of opinions to which they can have access in the nonsynchronized regime. As a consequence, highly connected nodes experience smaller values of local consensus, thus hindering the spreading dynamics. Therefore, there is a competition between two opposite effects (the existence of more spreading routes and the lack of local consensus) whose outcome is governed by the value of $\alpha$.

To shed light on this phenomenon, Fig. 4 reports the number of spreaders in the nonsynchronized regime $(\lambda=0)$ as a function of $(\beta, \alpha)$ for both SF-ER (solid lines) and SF-SF (dashed lines) configurations. Interestingly, for large values of $\alpha$, a small degree of consensus around hubs is enough to hinder their spreading ability, thus yielding a higher diffusion threshold than for the SF-ER multiplex. However, for small values of $\alpha$, the lack of local consensus around hubs becomes less relevant and the presence of highly connected nodes promotes ideas spreading, thus anticipating the diffusion threshold. This way, $\alpha$ can be interpreted as a kind of social pressure shaping the transmissibility of ideas as a function of their acceptance in society.

To further understand the role that $\alpha$ plays on the intertwined dynamics, we analyze in Fig. 5 the phase diagrams of the spreading dynamics varying this parameter as well as the average degree of the underlying SF-ER configuration. Note that the shift of the diffusion threshold as a consequence of the aforementioned interplay between topology and social pressure becomes more evident in this case. In addition, Fig. 5 highlights the role that social pressure plays on the emergence of abrupt or smooth transitions. Namely, increasing the social pressure over agents turns the emergence of spreaders and full 


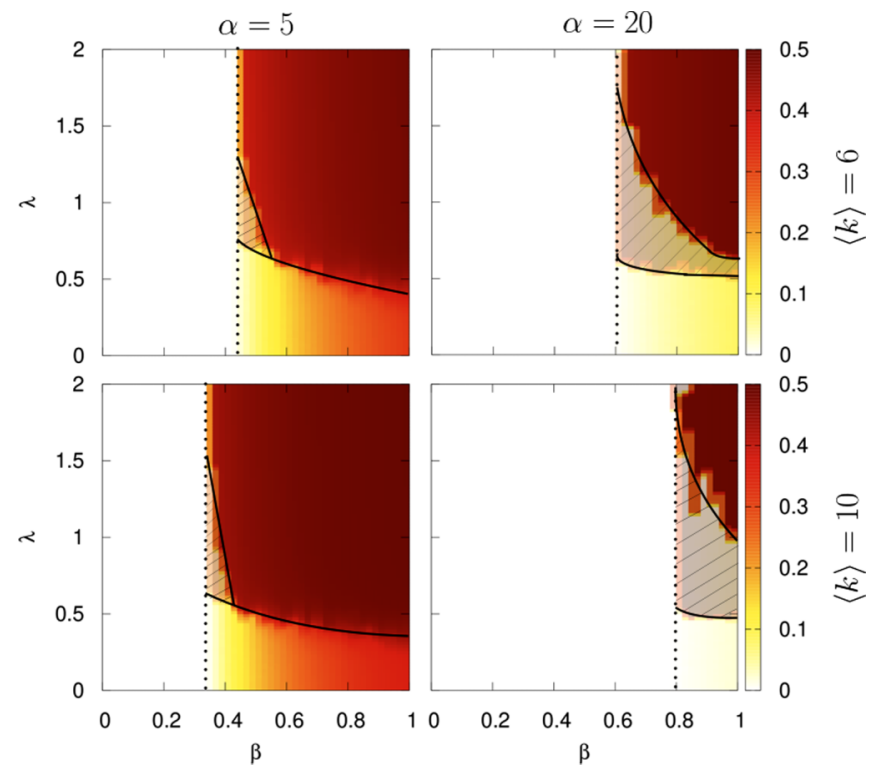

FIG. 5. Diagrams of the fraction of spreaders nodes $I$ (color code) as a function of the infectivity $\beta$ and of the coupling parameter $\lambda$ for SF-ER multiplex networks. The social pressure $\alpha$ is set to $\alpha=5$ (left panels) and $\alpha=20$ (right panels). The average degree of the ER spreading layer is fixed to $\langle k\rangle=6$ (top row) and to $\langle k\rangle=10$ (bottom row). In all panels, the fraction of spreaders has been obtained by averaging their values over $T=800$ steps. The striped regions in the panels highlight the parameter region $(\lambda, \beta)$ where hysteresis cycles appear due to the coexistence of two stable solutions: total consensus-disagreement in the synchronization layer and active-inactive spreaders at the information spreading layer. The black solid lines denote the critical values of the coupling $\lambda$ separating the two solutions.

consensus into an abrupt transition. In this sense, for $\alpha=5$, a macroscopic set of spreaders can emerge despite the lack of an unanimous opinion so that continuous transitions to the state of full consensus are more likely to occur. However, for $\alpha=20$, the diffusion of information is strongly hindered in the nonsynchronized regime. As a consequence, the existence of a macroscopic set of spreaders requires full consensus, thus leading to the emergence of discontinuous transitions.

Now, we characterize the influence of both dynamical and topological features on the emergence of spreaders. To this aim we put our focus on the nonsynchronized regime and study the value of the threshold $\beta_{c}$ for which the fraction of spreaders in the steady state is not negligible. This way, we show in Fig. 6(a) the dependence of $\beta_{c}$ on the social pressure $\alpha$ and the average degree of the diffusion layer $\langle k\rangle$. As anticipated above, the onset of an idea crucially depends on the interplay between both ingredients.

To shed more light on the role of each parameter, we show explicitly the evolution of $\beta_{c}$ as a function of $\langle k\rangle(\alpha)$ for several values of $\alpha(\langle k\rangle)$ in Figs. 6(b) and 6(c). Namely, Fig. 6(b) confirms that increasing the average degree yields a competition between the creation of more spreading routes and the decrease of the local consensus. This competition is clearly governed by the value of $\alpha$, so that for low $\alpha$ values, the local consensus loses relevance with respect to the increase of connections, thus leading to a lower value of $\beta_{c}$ as $\langle k\rangle$
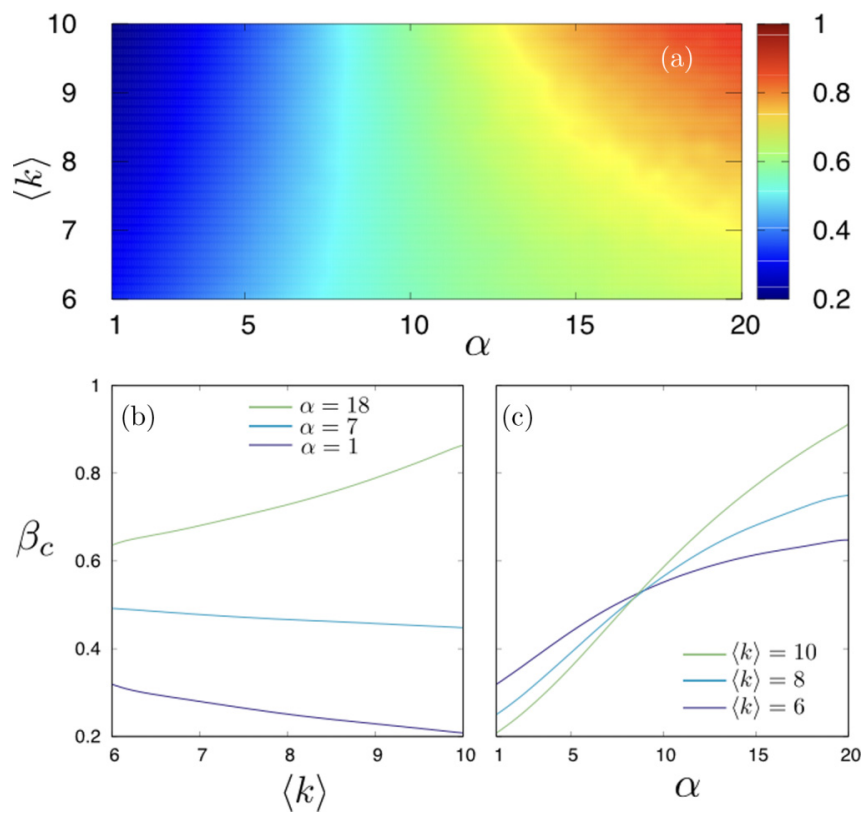

FIG. 6. (a) Diffusion threshold $\beta_{c}$ (color code) as a function of the social pressure $\alpha$ and the average degree $\langle k\rangle$ of the diffusion layer for the SF-ER configuration. In panels (b) and (c) we show the diffusion threshold $\beta_{c}$ as a function of the average degree $\langle k\rangle$ and the social pressure $\alpha$, respectively. In both cases we show these functions for several values of the social pressure $\alpha$ (b) and the average degree $\langle k\rangle(\mathrm{c})$.

increases. However, for high $\alpha$ values, the value of $\beta_{c}$ increases with $\langle k\rangle$ since spreaders emerge only when their neighbors share an unanimous opinion, i.e., when perfect consensus is established in their neighborhoods. Obviously, this condition is more difficult to achieve in the nonsynchronized regime as the number of connections (acquaintances) increases.

Finally, Fig. 6(c) reveals that reinforcing the social pressure always hinders the spread of ideas in the nonsynchronized regime. Interestingly, this effect is more evident as the average degree of individuals in the diffusion layer increases, i.e., the larger is the set of acquaintances of individuals, the more they are influenced by their social environment.

\section{CONCLUSIONS}

In this work we have introduced a model which allows us to study the interplay between information spreading and the emergence of consensus in a social system. To do so, we have considered a two-layer multiplex network in which one layer accounts for information spreading according to a SIS model, while the other layer describes the formation of consensus in a Kuramoto model. To couple the two dynamics, we have considered that the control parameters of each process depend on the dynamical state of the other process. Specifically, we assume that the two processes reinforce mutually, in such a way that the presence of many spreaders foster global consensus and the existence of similar opinions promotes their adoption.

Results of numerical simulations have revealed usual features about information spreading and consensus inside populations. For instance, we have characterized the limiting role 
of information spreading, since the absence of spreaders of an idea impedes the achievement of a global consensus on it among people with different initial opinions. In addition, we have also observed that the direct correlation between information spreading and the alignment of opinions leads to the onset of abrupt transitions and the appearance of bistability regions. These explosive transitions can have important consequences in real systems due to the drastic changes induced by perturbations in the bi-stability regions. Our findings add to recent studies devoted to determine the conditions leading to explosive phenomena in networks with a single layer [47-51] as well as in multilayer networks [29,52-54].

We have found that social pressure crucially governs the transitions leading to the emergence of the diffusion of ideas and consensus around them. Namely, for high social pressure values the multiplex undergoes an abrupt transition. This explosive onset happens when a macroscopic fraction of agents spread in one layer a widely accepted idea in the other one. The simultaneous activation of overlapping giant clusters has been also reported for other tightly coupled dynamics on multilayer networks. For instance, Baxter et al. [54] showed that abrupt percolation transitions appear as a result of the overlap between giant percolating clusters in all layers. However, when social pressure is relaxed overlapping clusters do not show up. As a result, the multiplex undergoes second-order transitions in the spreading layer while the consensus one remains inactive.

Finally, we have numerically studied the effects on the diffusion threshold of the interplay between the topology of the spreading layer and the local consensus around each individual. Interestingly, for high social pressure values, the diffusion of ideas is more hindered in heterogeneous networks than in homogeneous ones. Apparently, this is a counterintuitive and atypical result, since epidemiological models [3,55] usually predict smaller epidemic thresholds for heterogeneous networks. However, in this intertwined dynamics, the presence of hubs enhances the access to wider range of opinions. This leads to lower values of local consensus when these opinions are not aligned, thus inhibiting the propagation of information.

In a nutshell, the formalism introduced here constitutes a simple framework to characterize the mutual influence between the propagation of ideas in a population of agents and the alignment of their opinions. Our results point out that introducing positive correlations between the two processes leads to the emergence of explosive phenomena in both the spreading and the consensus dynamics, thus providing an alternative and multiplex-based way of creating abrupt transitions in models, such as the SIS and the Kuramoto model, usually showing continuous transitions.

\section{ACKNOWLEDGMENTS}

D.S.-P. acknowledges financial support from Gobierno de Aragón through a doctoral fellowship. Q.G. acknowledges financial support from China Scholarship Council (CSC) (Grant No. 201406020055). V.L. acknowledges support from the EPSRC Project No. EP/N013492/1. J.G.-G. and D.S.-P. acknowledge support from MINECO (Grants No. FIS201571582-C2-1 and No. FIS2017-87519-P), and Gobierno de Aragón/Fondo Europeo de Desarrollo Regional (FEDER) (Grant No. E36-17R to FENOL group).
[1] A. Arenas, A. Díaz-Guilera, J. Kurths, Y. Moreno and C. Zhou, Synchronization in complex networks, Phys. Rep. 469, 93 (2008).

[2] C. Castellano, S. Fortunato, and V. Loreto, Statistical physics of social dynamics, Rev. Mod. Phys. 81, 591 (2009).

[3] R. Pastor-Satorras, C. Castellano, P. Van Mieghem, and A. Vespignani, Epidemic processes in complex networks, Rev. Mod. Phys. 87, 925 (2015).

[4] D. J. Watts and S. H. Strogatz, Collective dynamics of smallworld networks, Nature 393, 440 (1998).

[5] R. Albert and A.-L. Barabási, Statistical mechanics of complex networks, Rev. Mod. Phys. 74, 47 (2002).

[6] M. E. J. Newman, The structure and function of complex networks, SIAM Rev. 45, 167 (2003).

[7] S. Boccaletti, V. Latora, Y. Moreno, M. Chavez, and D.-U. Hwang, Complex networks: Structure and dynamics, Phys. Rep. 424, 175 (2006).

[8] V. Latora, V. Nicosia, and G. Russo, Complex Networks: Principles, Methods and Applications (Cambridge University Press, Cambridge, 2017).

[9] R. Pastor-Satorras and A. Vespignani, Epidemic Spreading in Scale-Free Networks, Phys. Rev. Lett. 86, 3200 (2001).

[10] T. Nishikawa, A. E. Motter, Y.-C. Lai, and F. C. Hoppensteadt, Heterogeneity in Oscillator Networks: Are Smaller Worlds Easier to Synchronize? Phys. Rev. Lett. 91, 014101 (2003).
[11] J. Gómez-Gardeñes, Y. Moreno and A. Arenas, Paths to Synchronization in Complex Networks, Phys. Rev. Lett. 98, 034101 (2007).

[12] S. Funk, M. Salathé, and V. A. A. Jansen, Modelling the influence of human behavior on the spread of infectious diseases: A review, J. Roy Soc. Interface 7, 1257 (2010).

[13] C. T. Bauch and A. P. Galvani, Epidemiology: Social factors in epidemiology, Science 342, 4 (2013).

[14] S. Funk, E. Gilada, C. Watkinsb, and V. A. A. Jansen, Proc. Natl. Acad. Sci. USA 106, 6872 (2009).

[15] B. Steinegger, A. Cardillo, P. De Los Rios, J. Gómez-Gardeñes, and A. Arenas, Interplay between cost and benefits triggers nontrivial vaccination uptake, Phys. Rev. E 97, 032308 (2018).

[16] E. Bullmore and O. Sporns, Complex brain networks: Graph theoretical analysis of structural and functional systems, Nat. Rev. Neurosci. 10, 186 (2009).

[17] R. Criado, J. Flores, A. García del Amo, J. Gómez-Gardeñes, and M. Romance, A mathematical model for networks with structures in the mesoscale, Int. J. Comput. Math. 89, 291 (2012).

[18] M. De Domenico, A. Solé-Ribalta, E. Cozzo, M. Kivelä, Y. Moreno, M. A. Porter, S. Gomez, and A. Arenas, Mathematical Formulation of Multi-Layer Networks, Phys. Rev. X 3, 041022 (2013). 
[19] M. Kivelä, A. Arenas, M. Barthelemy, J. P. Gleeson, Y. Moreno, and M. A. Porter, Multilayer networks, J. Complex Netw. 2, 203 (2014).

[20] S. Boccaletti, G. Bianconi, R. Criado, C. Del Genio, J. GómezGardeñes, M. Romance, I. Sendiña-Nadal, Z. Wang, and M. Zanin, The structure and dynamics of multilayer networks, Phys. Rep. 544, 1 (2014).

[21] J. Gómez-Gardeñes, I. Reinares, A. Arenas, and L. M. Floría, Evolution of cooperation in multiplex networks, Sci Rep. 2, 620 (2012).

[22] S. Gómez, A. Díaz-Guilera, J. Gómez-Gardeñes, C. J. PérezVicente, Y. Moreno, and A. Arenas, Diffusion Dynamics on Multiplex Networks, Phys. Rev. Lett. 110, 028701 (2013).

[23] E. Cozzo, R. A. Baños, S. Meloni, and Y. Moreno, Contactbased social contagion in multiplex networks, Phys. Rev. E 88, 050801(R) (2013)

[24] C. Granell, S. Gomez, and A. Arenas, Dynamical Interplay between Awareness and Epidemic Spreading in Multiplex Networks, Phys. Rev. Lett. 111, 128701 (2013).

[25] L. V. Gambuzza, M. Frasca, and J. Gómez-Gardeñes, Intralayer synchronization in multiplex networks, Europhys. Lett. 110, 20010 (2015).

[26] Ch. I. del Genio, J. Gómez-Gardeñes, I. Bonamassa, and S. Boccaletti, Synchronization in networks with multiple interaction layers, Sci. Adv. 2, 1601679 (2016).

[27] R. Amato, N. E. Kouvaris, M. San Miguel, and A. Díaz-Guilera, Opinion competition dynamics on multiplex networks, New J. Phys. 19, 123019 (2017).

[28] A. Antonioni and A. Cardillo, Coevolution of Synchronization and Cooperation in Costly Networked Interactions, Phys. Rev. Lett. 118, 238301 (2017).

[29] V. Nicosia, P. S. Skardal, A. Arenas, and V. Latora, Collective Phenomena Emerging from the Interactions between Dynamical Processes in Multiplex Networks, Phys. Rev. Lett. 118, 138302 (2017)

[30] G. F. de Arruda, E. Cozzo, T. P. Peixoto, F. A. Rodrigues and Y. Moreno, Disease Localisation in Multilayer Networks, Phys. Rev. X 7, 011014 (2017).

[31] D. Soriano-Paños, L. Lotero, A. Arenas, and J. GómezGardeñes, Spreading Processes in Multiplex Metapopulations Containing Different Mobility Networks, Phys. Rev. X 8, 031039 (2018).

[32] A. Baronchelli, The emergence of consensus: A primer, R. Soc. Open Sci. 5, 172189 (2018).

[33] D. Strang and S. A. Soule, Diffusion in organizations and social movements: From hybrid corn to poison pills, Annual Review of Sociology 24, 265 (1998).

[34] J. Gómez-Gardeñes, L. Lotero, S. Taraskin, and F. Pérez-Reche, Explosive contagion in networks, Sci. Rep. 6, 19767 (2016).

[35] D. Centola and M. Macy, Complex contagions and the weakness of long ties, Amer. J. Sociol. 113, 702 (2007).

[36] I. Iacopini, G. Petri, A. Barrat, and V. Latora, Simplicial models of social contagion, Nat. Communs. 10, 2485 (2019).

[37] R. Axelrod, The dissemination of culture: A model with local convergence and global polarization, J. Conflict Resol. 41, 203 (1997).
[38] F. Battiston, V. Nicosia, V. Latora, and M. San Miguel, Layered social influence promotes multiculturality in the Axelrod model, Sci. Rep. 7, 1809 (2017)

[39] S. Boccaletti, J. A. Almendral, S. Guan, I. Leyva, Z. Liu, I. Sendiña-Nadal, Z. Wang, and Y. Zou, Explosive transitions in complex networks structure and dynamics: Percolation and synchronization, Phys. Rep. 660, 1 (2016).

[40] Y. Kuramoto, Self-entrainment of a population of coupled nonlinear oscillators, in International Symposium on Mathematical Problems in Theoretical Physics, edited by H. Araki, Lecture Notes in Physics Vol. 39 (Springer, Berlin, 1975).

[41] S. H. Strogatz, From Kuramoto to Crawford: Exploring the onset of synchronization in populations of coupled oscillators, Physica D 143, 1 (2000).

[42] J. A. Acebron, L. L. Bonilla, C. J. Perez Vicente, F. Ritort, and R. Spigler, The Kuramoto model: A simple paradigm for synchronization phenomena, Rev. Mod. Phys. 77, 137 (2005).

[43] S. Gómez, A. Arenas, J. Borge-Holthoefer, S. Meloni, and Y. Moreno, Discrete-time Markov chain approach to contact based diseases spreading in complex networks, Europhys. Lett. 89, 38009 (2010).

[44] B. Guerra and J. Gómez-Gardeñes, Annealed and mean-field formulations of disease dynamics on static and adaptive networks, Phys. Rev. E 82, 035101(R) (2010).

[45] S. Gómez, J. Gómez-Gardeñes, Y. Moreno, and A. Arenas, Nonperturbative heterogeneous mean-field approach to epidemic spreading in complex networks, Phys. Rev. E 84, 036105 (2011).

[46] A.-L. Barabási and R. Albert, Emergence of scaling in random networks, Science 286, 509 (1999).

[47] J. Gómez-Gardeñes, S. Gómez, A. Arenas, and Y. Moreno, Explosive Synchronization Transitions in Scale-Free Networks, Phys. Rev. Lett. 106, 128701 (2011).

[48] D. Achlioptas, R. M. D’Souza, and J. Spencer, Explosive percolation in random networks, Science 323, 1453 (2009).

[49] A. E. Motter, S. A. Myers, M. Anghel, and T. Nishikawa, Spontaneous synchrony in power-grid networks, Nat. Phys. 9, 191 (2013).

[50] S. V. Buldyrev, R. Parshani, G. Paul, H. E. Stanley, and S. Havlin, Catastrophic cascade of failures in interdependent networks, Nature 464, 1025 (2010).

[51] X. Zhang, Y. Zou, S. Boccaletti and Z. Liu, Explosive synchronization as a process of explosive percolation in dynamical phase space, Sci. Rep. 4, 5200 (2014).

[52] X. Zhang, S. Boccaletti, S. Guan, and Z. Liu, Explosive Synchronization in Adaptive and Multilayer Networks, Phys. Rev. Lett. 114, 038701 (2015).

[53] M. M. Danziger, I. Bonamassa, S. Boccaletti, and S. Havlin, Dynamic interdependence and competition in multilayer networks, Nat. Phys. 15, 178 (2019).

[54] G. J. Baxter, S. N. Dorogovtsev, A. V. Goltsev, and J. F. F. Mendes, Avalanche Collapse of Interdependent Networks, Phys. Rev. Lett. 109, 248701 (2012).

[55] M. J. Keeling, K. T. Eames, Networks and epidemic models, J. Roy. Soc. Interface 2, 295 (2005). 